\title{
The journey towards elimination of gambiense human African trypanosomiasis: not far, nor easy
}

\author{
J. R. FRANCO ${ }^{1}$, P. P. SIMARRO ${ }^{1}$, A. DIARRA ${ }^{2}$, J. A. RUIZ-POSTIGO ${ }^{3}$ and J. G. JANNIN ${ }^{1}$ \\ ${ }^{1}$ World Health Organization, Control of Neglected Tropical Diseases, Innovative and Intensified Disease Management, \\ 20, Av. Appia, 1211 Geneva, Switzerland \\ ${ }^{2}$ World Health Organization, WHO Inter Country Support Team for Central Africa, Regional Office for Africa, IST/CA, \\ BP 820, Libreville, Gabon \\ ${ }^{3}$ World Health Organization, Communicable Disease Control, Control of Tropical Diseases and Zoonoses, Regional Office for \\ the Eastern Mediterranean, P.O. Box: 7608, Nasr City, Cairo 11371, Egypt
}

(Received 4 October 2013; revised 19 and 20 November 2013; accepted 21 November 2013; first published online 28 fanuary 2014)

SUMMAR Y

Considering the epidemic situation of gambiense human African trypanosomiasis (HAT) at the end of the twentieth century, the World Health Organization (WHO) and partners strengthened disease control and surveillance. Over the last 15 years, the activities implemented through the National Control Programmes have brought gambiense HAT under control and now its elimination is deemed as an achievable goal. In 2012, WHO targeted gambiense HAT for elimination as a public health problem by 2020 . The final goal will be the sustainable disease elimination by 2030 , defined as the interruption of the transmission of gambiense HAT. The elimination is considered feasible, because of the epidemiological vulnerability of the disease, the current state of control, the availability of strategies and tools and international commitment and political will. Integration of activities in the health system is needed to ensure the sustainability of the elimination. The development of user-friendly diagnostic and treatment tools will facilitate the integration process. Adequate funding is needed to implement activities, but also to support research that will make the elimination sustainable. A long-term commitment by donors is needed and ownership of the process by endemic countries is critical.

Key words: Human African trypanosomiasis, HAT, sleeping sickness, disease elimination, gambiense HAT, T.b. gambiense.

\section{BACKGROUND}

Human African trypanosomiasis (HAT), or sleeping sickness, is a disease caused by extracellular protozoa belonging to the genus Trypanosoma, which are transmitted by the bite of an infected tsetse fly (genus: Glossina). The disease is endemic in 36 countries in sub-Saharan Africa, with about 70 million people living at risk of infection (Simarro et al. 2012). HAT is considered as a neglected tropical disease (NTD) (World Health Organization, 2012a) and it afflicts mainly neglected populations in rural areas where suitable environmental conditions for the occurrence of the tsetse vector are present.

There exist two human pathogen subspecies (i.e. Trypanosoma brucei gambiense and Trypanosoma brucei rhodesiense), which are morphologically indistinguishable. Western and central sub-Saharan Africa are affected by $T$. b. gambiense, which leads to a chronic disease that may last for years (gambiense

* Corresponding author: World Health Organization, Control of Neglected Tropical Diseases, Innovative and Intensified Disease Management, 20, Av. Appia, 1211 Geneva, Switzerland. E-mail: francoj@who.int
HAT) (Checchi et al. 2008). This form of HAT currently accounts for more than $95 \%$ of the total cases. In eastern and southern Africa T. $b$. rhodesiense causes an acute disease that may be fatal within weeks or months (rhodesiense HAT) (World Health Organization, 1998; Brun et al. 2010).

After inoculation, the parasite is initially found in the lymphatic system and bloodstream (haemolymphatic stage) but after a period of variable duration, trypanosomes cross the blood-brain barrier and enter the central nervous system (meningoencephalic stage) causing a progressive neurological breakdown. If untreated, patients progress to coma, severe organ failure, and eventually death (Kennedy, 2008).

HISTORICAL PROGRESS, SITUATION DURING THE PAST DECADE AND CURRENT EPIDEMIOLOGICAL STATUS OF THE DISEASE

After the epidemics that took place at the beginning of the 20th century, which killed an estimated 300000 and 500000 people in the Congo Basin and the Busoga focus, respectively (Hide, 1999; Steverding, 2008), it appeared that HAT had been progressively 


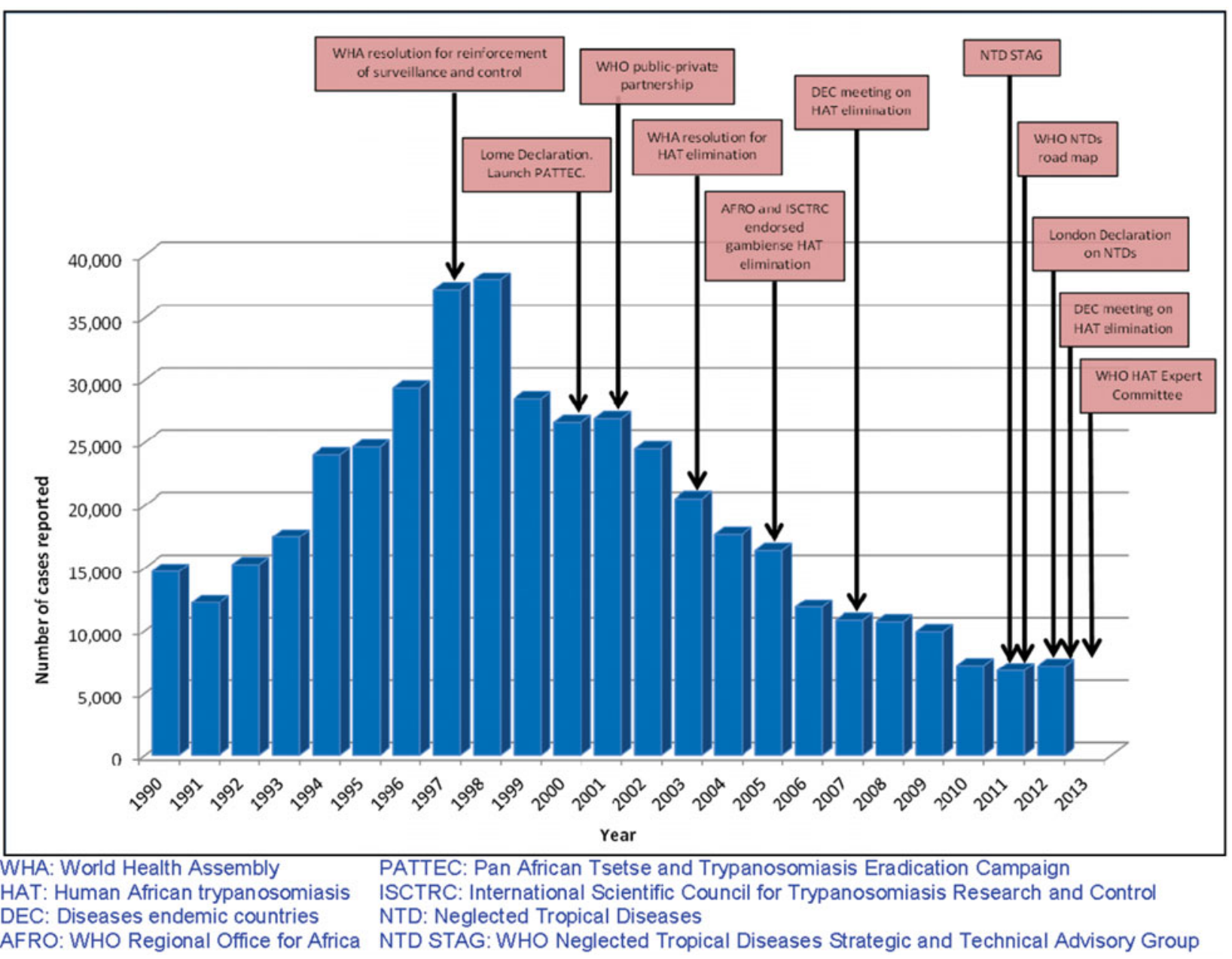

Fig. 1. Number of gambiense HAT new cases reported (1990-2012) and key events on the road to the elimination.

controlled during the mid-50s. The annual number of new declared cases decreased from more than 55000 (1940) to a minimum of 4435 (1964) (World Health Organization, 2000). In the following decades the rarity of the disease and competition from other health priorities led to neglect of disease control and surveillance. This allowed the number of new cases to rise again to alarming levels at the end of the twentieth century, reaching 37991 new cases declared in 1998 (World Health Organization, 2006), with an estimation of more than 300000 people infected (World Health Organization, 1998). In addition, social instability in some endemic countries and the scarcity of funds contributed to hampering disease control.

To face this epidemic situation, the World Health Organization (WHO) and partners implemented a series of initiatives aimed at bringing HAT back under control. In 2001, pharmaceutical companies producing medicines against HAT (Aventis (currently Sanofi) and Bayer) agreed to provide these free of charge to affected countries through the WHO. In addition, resources were made available for strengthening disease control and surveillance through the support of the WHO to Sleeping Sickness National
Control Programmes (SSNCP). At the same time, bilateral cooperation (Belgium, France) and nongovernmental organizations (NGOs) joined the effort and contributed to reversing the upward trend in the number of HAT cases (Simarro et al. 2011). These enhanced control activities resulted in an improved epidemiological knowledge of the disease, as well as in a sustained decrease in the number of reported cases. By 2009, the number of reported new cases had fallen below 10000 , with fewer than 8000 in 2010, 2011 and 2012, and an estimated number of infections at around 20000 (World Health Organization, 2013c).

Recent trends in new HAT cases (Fig. 1) are promising, but figures should be interpreted with care: the disease occurs mainly in remote rural areas where health infrastructures are weak. Cases are not always recognized, which results in significant underdetection and under-reporting. Under-detection and under-reporting are difficult to estimate, but some available data suggest that $50-65 \%$ of cases are currently not reported (Mumba et al. 2011; Simarro et al. 2013a). In particular, some of the areas concerned by this problem are hard to reach because of insecurity or difficult terrain. 
It has been estimated that 70 million people live at different levels of risk of HAT infection. 57 million $(81 \%)$ are at risk of gambiense HAT, and this population is distributed over an area of approximately 1.38 million $\mathrm{km}^{2}$ in 14 of the 24 countries listed as endemic for gambiense HAT. More than 2 million people live in areas classified as high to very high risk of gambiense HAT (Simarro et al. 2012). The distribution of the disease, the population and areas at risk and the level of control activities are shown in Table 1.

The number of new reported cases of gambiense HAT has been below 10000 since 2009, with 7106 cases declared in 2012. As of 2008, gambiense HAT cases represent $98 \%$ of the total HAT cases and cases of rhodesiense HAT accounting for the remaining $2 \%$. In the last 5 years (2008-2012), 78\% of gambiense HAT cases were reported from the Democratic Republic of the Congo (DRC), followed by Central African Republic (8\%), South Sudan (4\%), Chad (3.5\%) and Angola (3\%) (Table 1) (World Health Organization, 2012b).

Currently, SSNCP are functional in 15 endemic countries (Angola, Benin, Burkina Faso, Cameroon, Central African Republic, Chad, Congo, Côte d'Ivoire, the DRC, Equatorial Guinea, Gabon, Guinea, Mali, Uganda and Togo). All these countries have a functioning surveillance system for gambiense HAT. In Ghana, Nigeria and South Sudan there is not a structured SSNCP but there is a surveillance system run by different structures of the Ministry of Health. In Gambia, Guinea Bissau, Liberia, Niger, Senegal and Sierra Leone, SSNCP do not exist, and no surveillance activities are performed. Field assessments of the epidemiological situation have been carried out in Senegal and Sierra Leone (Table 1).

\section{EPIDEMIOLOGICAL CHARACTERISTICS OF DIFFERENT FOCI OF GAMBIENSE HAT}

HAT has a patchy geographical distribution, which is restricted to circumscribed areas (foci) (Welburn and Maudlin 2012). This distributional pattern is caused by a complex set of factors involving interactions between the environment, the host, the vector and the parasite. These complex relationships are not always fully understood. The foci of the disease have been fairly stable over time. The focus was qualitatively defined by the WHO Expert Committee in 1986 as 'a zone of transmission to which a geographical name is given (locality, region or river)' (World Health Organization, 1986). This definition is useful to allocate resources, to plan control activities, to measure the burden of the disease and to monitor the evolution of the epidemiological situation. Currently, 303 foci of gambiense HAT are identified (Table 1) (World Health Organization, 2012b).

Analysing the epidemiological patterns of these foci, it is possible to distinguish three different situations. The three epidemiological settings can be characterized on the basis of the reported intensity of disease transmission (Simarro et al. 2012, 2013a):

1. Foci at high to very high intensity of transmission. These are defined as those where the average annual number of cases reported is at least 1 per 1000 inhabitants.

2. Foci at moderate intensity of transmission. They are those where at least 1 case per 10000 inhabitants (but less than 1 per 1000 inhabitants) has been reported yearly.

3. Foci at low to very low intensity of transmission. They are those where at least 1 case per 1000000 inhabitants per annum (but less than 1 case per 10000 inhabitants) has been observed.

The average annual number of reported cases can be calculated for different time periods. In previous studies, a 10-year study period was used. However, a shorter time period (i.e. 5 years) might be more appropriate to follow the evolution of the epidemiological situation.

There are still a few areas where transmission intensity is not well known, and which require further investigation. Among these areas we distinguish two different situations:

1. Foci where disease transmission is known to be taking place (cases are present), but which are characterized by difficult access because of terrain or security constraints. As surveillance in these areas has been hampered, the intensity of transmission needs to be better assessed.

2. Foci which have not reported cases in the last decades but which do not have an effective surveillance system in place. These areas have a history of disease transmission, and for most of them the current ecological characteristics still make it possible. The absence of reported cases over a long period of time makes the absence of transmission probable. Investigations are needed to ascertain the presence or absence of transmission.

THE CASE OF RHODESIENSE HAT

Rhodesiense HAT has to be considered as a zoonosis with occasional presence in human beings. Its current epidemiology is characterized by a low level of endemicity with sporadic human cases. However, epidemics can occur. They have been linked to environmental changes or major social disruptions (Morris, 1959; Fèvre et al. 2001; Jelinek et al. 2002; Kaare et al. 2007), which have resulted in changes in population distribution and large-scale movements of livestock and people to marginal areas more exposed to infected tsetse flies. As opposed to 
Table 1. Gambiense HAT endemic countries (cases reported, area and population at risk, number of foci, surveillance and control activities)

\begin{tabular}{|c|c|c|c|c|c|c|c|c|c|c|}
\hline \multirow[b]{2}{*}{ Country } & \multicolumn{2}{|c|}{ No. of cases } & \multirow{2}{*}{$\begin{array}{l}\text { Area at risk } \\
\left(\mathrm{km}^{2} \times 10^{2}\right) \\
(\% \text { of total } \\
\text { country area })\end{array}$} & \multirow{2}{*}{$\begin{array}{l}\text { Pop. at risk } \\
\left(\text { no. people } \times 10^{3}\right) \\
(\% \text { of total country } \\
\text { population })\end{array}$} & \multirow{2}{*}{$\begin{array}{l}\text { No. of foci } \\
\text { described }\end{array}$} & \multicolumn{5}{|c|}{ Activities of surveillance and control } \\
\hline & $2003-2007$ & 2008-2012 & & & & SSNCP & Active screening & Passive screening & Vector control & Surveillance \\
\hline Angola & 8875 & 1206 & $2253(18 \%)$ & $5864(46 \%)$ & 46 & Yes & Yes & Yes & Sporadic & Yes \\
\hline Benin & 0 & 0 & - & - & 3 & Yes & No & Integrated & No & Yes \\
\hline Burkina Faso & 0 & 0 & - & - & 5 & Yes & Sporadic & Yes & Yes & Yes \\
\hline Cameroon & 75 & 75 & $173(4 \%)$ & $631(3 \%)$ & 12 & Yes & Yes & Yes & Sporadic & Yes \\
\hline $\begin{array}{l}\text { Central African } \\
\text { Republic }\end{array}$ & 3057 & 3156 & $658(10 \%)$ & $433(10 \%)$ & 8 & Yes & Yes & Yes & No & Yes \\
\hline Chad & 1268 & 1411 & $142(1 \%)$ & $465(5 \%)$ & 4 & Yes & Yes & Yes & Sporadic & Yes \\
\hline Congo & 2477 & 456 & $1150(34 \%)$ & $2566(64 \%)$ & 19 & Yes & Yes & Yes & No & Yes \\
\hline Côte d'Ivoire & 226 & 49 & $286(9 \%)$ & $2672(13 \%)$ & 15 & Yes & Yes & Yes & Sporadic & Yes \\
\hline $\begin{array}{l}\text { Democratic } \\
\text { Republic of the } \\
\text { Congo }\end{array}$ & 48304 & 31716 & $7894(34 \%)$ & $36242(53 \%)$ & 118 & Yes & Yes & Yes & Sporadic & Yes \\
\hline Equatorial Guinea & 90 & 29 & $65(24 \%)$ & $43(7 \%)$ & 4 & Yes & Yes & Yes & Sporadic & Yes \\
\hline Gabon & 189 & 86 & $167(6 \%)$ & $803(53 \%)$ & 8 & Yes & Yes & Yes & Sporadic & Yes \\
\hline Gambia & 0 & 0 & - & - & 1 & No & No & No & No & No \\
\hline Ghana & 0 & 0 & - & - & 1 & No & No & Yes & Yes & Yes \\
\hline Guinea & 436 & 364 & $184(8 \%)$ & $2606(26 \%)$ & 13 & Yes & Yes & Yes & Yes & Yes \\
\hline Guinea Bissau & 0 & 0 & - & - & 3 & No & No & No & No & No \\
\hline Liberia & 0 & 0 & - & - & 4 & No & No & No & No & No \\
\hline Mali & 0 & 0 & - & - & 6 & Yes & No & Yes & Yes & Yes \\
\hline Niger & 0 & 0 & - & - & 1 & No & No & No & No & No \\
\hline Nigeria & 65 & 7 & $70(1 \%)$ & $2183(2 \%)$ & 4 & No & Sporadic & No & Sporadic & Yes \\
\hline Senegal & 0 & 0 & - & - & 5 & No & No & No & No & $\begin{array}{l}\text { Field } \\
\text { assessment }\end{array}$ \\
\hline Sierra Leone & 0 & 0 & $18(3 \%)$ & $170(3 \%)$ & 5 & No & No & No & No & $\begin{array}{l}\text { Field } \\
\text { assessment }\end{array}$ \\
\hline South Sudan & 7914 & 1785 & $1001(16 \%)$ & $1270(18 \%)$ & 9 & No & Sporadic & Yes & No & Yes \\
\hline Uganda & 1616 & 459 & $176(9 \%)$ & $2131(7 \%)$ & 7 & Yes & Yes & Yes & Sporadic & Yes \\
\hline Togo & 0 & 0 & - & - & 2 & Yes & No & Integrated & No & Yes \\
\hline Total & 74592 & 40799 & $14239(10 \%)$ & $58080(13 \%)$ & 303 & & & & & \\
\hline
\end{tabular}

SSNCP: Sleeping Sickness National Control Programme. 
gambiense HAT, the possibility of importation of rhodesiense HAT into new areas is not negligible (Fèvre et al. 2004). The non-human reservoir is a key element in the transmission cycle of rhodesiense HAT (Hide et al. 1996), and the control of the disease requires a multisectoral approach where animal health and natural resources management (national parks and game reserves) have an important role to play (Selby et al. 2013).

Therefore, being a zoonosis with an important animal reservoir, the elimination of rhodesiense HAT as the total interruption of transmission of the disease is considered not to be feasible and for this reason has not been included in this analysis. However, the possibility of its elimination has been considered as a public health problem (World Health Organization, 2012a).

KEY EVENTS AND DECLARATIONS ON THE ROAD TOWARDS ELIMINATION OF GAMBIENSE HAT

Over the last 15 years, the success in bringing gambiense HAT from an epidemic situation to control has been accompanied by a number of political and technical declarations. The commitment from partners has also contributed to bring about a situation where the elimination of gambiense HAT is now considered as a reachable goal (Fig. 1). Along the way, key milestones have included:

In 1997, concerned by the worrying epidemiological situation of HAT, the 50th World Health Assembly (WHA) urged all member states in endemic areas to reinforce control and surveillance activities and coordinate their actions through the Programme Against African Trypanosomiasis PAAT (a joint initiative of the Food and Agriculture Organization of the United Nations - FAO, the International Atomic Energy Agency - IAEA, the WHO and the African Union - Interafrican Bureau of Animal Resources - AU/IBAR)) and emphasized the need for resources to expand the control and surveillance of the disease (World Health Organization, 1997).

In 2000, during the summit held in Lomé (Togo), the heads of states and governments of African countries, considering the severe impact of tsetse-transmitted trypanosomiasis on the socio-economic development, expressed their political will to embark on a continental campaign of eradication, and consequently the African Union (AU) established the Pan African Tsetse and Trypanosomiasis Eradication Campaign (PATTEC).
In 2001, two manufacturers of medicines against HAT, Aventis and Bayer, committed to ensuring production of the disease-specific drugs and to donating the drugs to the WHO for free distribution. An agreement was signed between Aventis and WHO aimed at providing the WHO with the financial support to face the worrying situation of gambiense HAT.

In 2003, the 56th WHA welcomed and supported the PATTEC initiative and commended the efforts of the WHO to implement a programme for the elimination of African trypanosomiasis as a public health problem (World Health Organization, 2003).

In 2004, the 57th WHA Assembly urged member states 'to continue to give high priority to the control of HAT' and requested the WHO to refine control strategies and to promote an integrated approach with the sectors and agencies concerned (World Health Organization, 2004).

In 2005, the goal of elimination of HAT was endorsed by the 55th WHO Regional Committee for Africa and by the International Scientific Council for Trypanosomiasis Research and Control (ISCTRC).

In 2006 the agreement between WHO and SanofiAventis (previously Aventis) was renewed. The goal was to consolidate the control of the disease, preparing the conditions for elimination and to replace melarsoprol as the first-line treatment for the second stage of gambiense HAT.

In 2007, representatives of countries in which HAT is endemic gathered in Geneva (Switzerland) to discuss the elimination of HAT as a public health problem. They concluded that the elimination of gambiense HAT was feasible (World Health Organization, 2012b).

In 2011, the WHO-Sanofi (previously SanofiAventis) agreement was renewed with the goal of implementing strategies to achieve and sustain disease elimination through innovative strategies. In the same year, the WHO's Strategic and Technical Advisory Group for NTDs (WHO NTD STAG) deemed elimination to be technically feasible and HAT was included in the WHO Roadmap on NTDs, which was launched in early January 2012 with the target date for elimination as a public health problem set by 2020 (World Health Organization, 2012b).

In January 2012 a number of stakeholders including pharmaceutical companies, donors and some NGOs committed to the largest coordinated effort against NTDs through the London Declaration (World Health Organization, 2013a). The declaration 
supports the WHO Roadmap on NTDs to sustain, expand and extend programmes to eliminate, among other NTDs, HAT by 2020.

In December 2012, WHO convened a meeting of SSNCPs focal points, experts from WHO collaborating centres and members of the WHO STAG NTD in Geneva. Elimination of gambiense HAT was endorsed by SSNCPs of diseaseendemic countries. Strategies, tools and criteria for the process of eliminating gambiense HAT were laid out.

In April 2013, the WHO Expert Committee on HAT control and surveillance met in Geneva after 18 years, to discuss the current epidemiological patterns of the disease, the new diagnostic approaches and the new therapeutic regimens. Final recommendations to achieve disease elimination were addressed (World Health Organization, 2013c).

\section{CONCEPT OF ELIMINATION OF HAT}

The WHO Roadmap on NTDs included HAT with the target date for elimination as a public health problem by 2020 , defined as the reduction of HAT incidence to fewer than 1 new case per 10000 population in at least $90 \%$ of foci and to fewer than 2000 cases reported globally (World Health Organization, 2012a). The disease-endemic countries and experts from WHO collaborating centres considered this target as an advanced level of control only and as an intermediate objective in the way to reach the final goal of sustainable HAT elimination by 2030 . This last goal was defined following the WHO NTD STAG recommendation as the interruption of the transmission of gambiense HAT (zero disease incidence) in a condition where continued actions to prevent re-emergence of the diseases may be required (World Health Organization, 2012b).

\section{FEASIBILITY OF ELIMINATION OF GAMBIENSE} HAT

In 1989, the International Task Force for Disease Eradication (ITFDE) set out criteria for assessing potential eradication or elimination of diseases or conditions (Centers for Disease Control and Prevention, 1993), some related to the technical feasibility (epidemiological vulnerability, availability of effective and practical interventions or demonstrated feasibility of elimination) and others related to the political will or popular support as the perceived burden of the disease.

\section{Epidemiological vulnerability}

Gambiense HAT does not spread easily and humans are the only significant reservoir. Animal hosts have been described but more data are needed to elucidate their role as a reservoir in the transmission cycle. Such a role does not seem to be major (Funk et al. 2013).

The geographic distribution of the disease is well known and limited to 24 endemic countries. Besides, $97 \%$ of the cases are declared in just 5 countries, with approximately $80 \%$ of all the cases being reported from the DRC (Simarro et al. 2013a). In addition, in countries where gambiense HAT is endemic, only $10 \%$ of the national area is affected by the transmission of the disease (Simarro et al. 2012). A comprehensive database (WHO Atlas of HAT) compiles geo-referenced data which enable mapping at the village level, cases and screening activities reported from 2000 (Simarro et al. 2010).

\section{Effective, practical intervention tools available}

There is no vaccine against trypanosomiasis, and one will not become available in the coming years. However, effective tools for screening and treatment are available, although they are not easy to implement. Also, control tools are not appropriate for the involvement of fixed facilities of the health system in control and surveillance. Nevertheless, these tools have proven effective in reducing prevalence. The production and quality of specific medicines is guaranteed and they are affordable as they are generously donated at no cost by manufacturers. Availability is ensured by an effective distribution system managed by the WHO in collaboration with MSF-Logistique.

Integration of treatment and diagnosis in the health system is of the essence to spread the activities in time and space and ensure sustainability. Cheaper and user-friendlier diagnosis and treatment tools will make integration easier. There are currently new promising screening (Buscher et al. 2013) and treatment tools (Torreele et al. 2010; Jacobs et al. 2011) in the pipeline, which could facilitate the involvement of the health system.

Complete elimination of the vector does not seem feasible (World Health Organization, 1998; Molyneux, 2001; Hargrove, 2002). Nevertheless, new tools for vector control are being introduced, which are cheaper and easier to use (Esterhuizen et al. 2011). They have a role to play in supporting targeted interventions in particular transmission areas, where vector control will synergize with medical approaches.

\section{Demonstrated feasibility of elimination}

As a proof of principle, elimination has been achieved previously in some foci (Bruto da Costa et al. 1916; Simarro et al. 2006) and a strong reduction of the disease was reached at the continental level in the past 
with tools that have lower performance levels than those available today (Simarro et al. 2008; Welburn et al. 2009). Technical feasibility was acknowledged by SSNCPs during a meeting held in Geneva in December 2012 (World Health Organization, 2012b).

\section{Political will}

Political commitment to fight the disease was expressed by the heads of state and government of African countries through an official declaration issued from the summit of the OAU held in Lomé in 2000 (Organization of African Unity, 2001). In addition, WHO member states committed to HAT elimination by endorsing the WHO Road Map on NTD during the 66th WHA held in Geneva in May 2013 (World Health Organization, 2012a, 2013b). The international community showed their commitment to HAT elimination through the London Declaration in January 2012 (World Health Organization, 2013c).

\section{Perceived burden of the disease}

The disease has a case fatality rate that is close to $100 \%$. In endemic areas, people fear infection because of the suffering, lethality and the risk of stigma it entails. Affecting mainly individuals of productive age, and being a chronic disease, a case of gambiense HAT in a family unit causes important economic losses, as measured in terms of decreased income and increased expenditure (Lutumba et al. 2007).

\section{STRATEGIES FOR ELIMINATION OF GAMBIENSE} HAT

In the case of gambiense HAT, three classical control methods have been described and used for almost 100 years:

1. Active case-finding by mobile teams, which perform a serological screening in large populations (villages, neighbourhoods or other population settlements) and subsequently seek parasitological confirmation of serological suspects. Active case detection may determine a substantial reduction in disease prevalence, mainly when applied in successive rounds (Robays et al. 2004; Simarro et al. 2006; Checchi et al. 2012). Low participation rates of at-risk populations have been described under certain circumstances mainly when screenings are periodically repeated, prevalence is lowering, and the impact of the active case finding is decreasing (Robays et al. 2004; Welburn et al. 2009). Active case finding always has to include adequate treatment of all detected cases.
2. Passive case-finding and adequate treatment by fixed health facilities. This method concerns patients seeking healthcare with signs or symptoms suggestive of HAT infection. Serological tests are used for initial screening, followed by tests for parasitological confirmation in serological suspects. The introduction of individual and simple serological tests (lateral flow formats) (Buscher et al. 2013) can facilitate passive screening in sites where staff are not highly skilled in HAT-related techniques. Cases should always be adequately treated after detection.

3. Vector control to reduce the tsetse population, using the different methods available according to the characteristics of the different foci (World Health Organization, 2013c).

To combat the disease it is necessary to combine these three methods as appropriate. In the different epidemiological situations, different combinations and 'dosage' of each method has to be deployed, based on:

- Intensity of transmission;

- Precise understanding of the epidemiological setting, including geographic and demographic data;

- Accessibility and capabilities of the existing health facilities; and

- Knowledge of vector ecology, including the patterns of human-vector contact, the sites where vector control must be applied and the methods to be utilized.

Control and elimination strategies defined according to the intensity of transmission and epidemiological settings have to be flexible and dynamic enough to be adapted to the changing disease patterns and capacities of the local health services (Simarro et al. 2013a).

IMPLEMENTATION OF CONTROL AND SURVEILLANCE ACTIVITIES ACCORDING TO THE EPIDEMIOLOGICAL STATUS OF EACH VILLAGE

Considering that HAT is a focal disease, the focus and its level of transmission is the epidemiological unit and disease elimination strategies must be planned and monitored at the focus rather than the country level. At the same time, field interventions are to be planned and implemented at the village level, according to its specific epidemiological status.

Villages in each focus can be categorized according to the presence of cases in the previous years:

Villages which have reported cases in the previous 3 years.

Villages which have reported cases in the previous 5 years, but not in the previous three. 


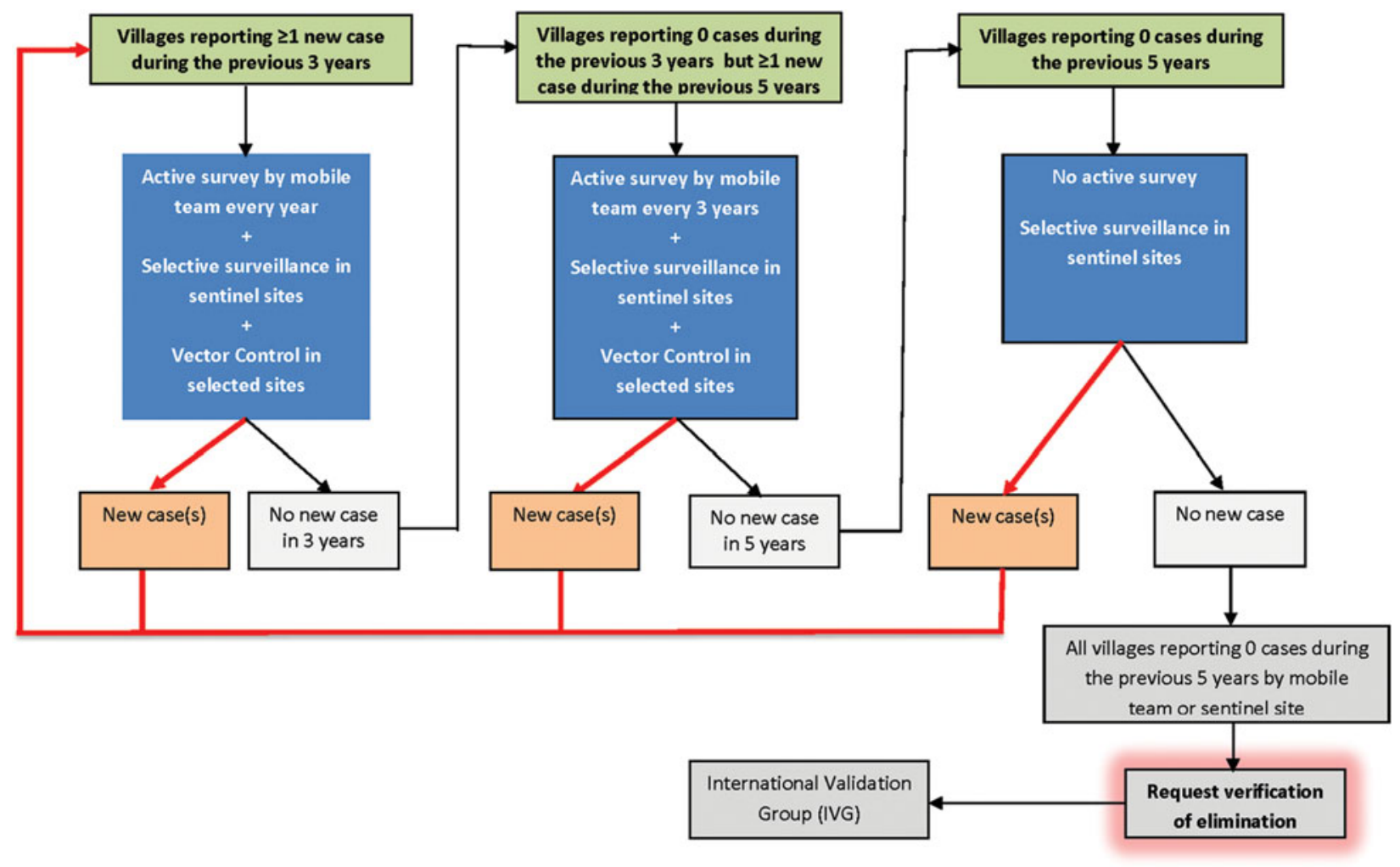

Fig. 2. Algorithm of interventions according to the epidemiological status of the villages in each focus.

Villages which have reported no cases in the previous 5 years.

Control and surveillance activities therefore will be planned according to this village-level categorization within a focus (Fig. 2). The classification of villages is updated yearly. To this end, diagnosed cases have to be carefully reported, including the possible village where the transmission took place.

\section{Active screening}

Active screening plays an important role in foci where transmission is high or very high. The active casefinding through mobile teams will be done yearly in all the villages which have presented cases in the previous 3 years. In the villages which have reported cases in the previous 5 years, but not in the previous 3 , an active screening will be performed every 3 years. In foci at moderate, low and very low intensity of transmission it is expected that the number of villages presenting cases will be low, and therefore the volume of active screening will be limited. The Card Agglutination Test for Trypanosomiasis (CATT) is the main tool used for active screening.

If a serological suspect detected in a sentinel centre is confirmed by parasitological examinations, a reactive active case-finding survey will be carried out for 3 consecutive years in the area of activity of the newly detected case. In some foci, the skills of the local health staff to perform the parasitological tests and an active screening could have been lost; in those cases, support from national or regional institutions can be requested in order to carry out the active screening and to build the local capacity.

\section{Passive screening}

Passive screening has to be performed in the selected health structures located within the focus (sentinel sites). The sentinel sites will be selected according to their capacities, the population covered and the frequenting by the population at risk. The performance of the passive detection could be increased by healthcare workers in health posts, who could detect the signs and symptoms evoking HAT infection and then refer clinical suspects to the sentinel sites.

Passive screening is the most important component for gambiense HAT control and surveillance in foci with low and very low intensity of transmission, where infection is rare and active case detection has poor cost-effectiveness. Passive screening is also a key element in high, very high and moderate transmission foci, as effectiveness of active screening is limited because of incomplete attendance at screening sessions, inherent limitations of screening tools and the lag between successive surveys.

If serological positive cases are detected by sentinel sites among clinical suspects through individual screening tests (also erroneously called rapid diagnostic tests - RDT), parasitological tests have to be carried out to confirm the diagnosis. If the serological 
suspicion is not confirmed, in particular epidemiological settings samples could be sent to a WHO collaborating centre for a more specific test (i.e. immune trypanolysis) for confirming the presence of anti-trypanosomal antibodies (Van Meirvenne et al. 1995; Jamonneau et al. 2010).

When staff at the peripheral level are not familiar with parasitological tests for confirmation, serological suspects could be referred for parasitological tests to health structures able to perform them or request support from national or regional referral centres.

\section{Vector control}

Vector control is an efficient tool to reduce tsetse flies numbers and therefore to reduce HAT transmission (Solano et al. 2013). Vector control is applied according to medical results in selected sites. It could play an important role in high-prevalence foci to help reduce transmission quickly, or in foci where the intensity of transmission does not decrease after repeated active screening rounds. It is not a priority in foci with low or very low intensity transmission, where it is only applicable if the intensity of transmission is not reduced after repeated active screening rounds.

There are a variety of tools for tsetse control and the choice of the most suitable will depend on local conditions (World Health Organization, 2013c).

MONITORING AND EVALUATING GAMBIENSE HAT ELIMINATION. CERTIFICATION AND

VALIDATION OF ELIMINATION

Continuous monitoring and evaluation of the HAT elimination process is needed to adapt the strategies to the epidemiological situation and to assess the results obtained. It will assure the sustainability of the elimination and will prevent the re-emergence of the disease.

The monitoring and evaluation of progress toward elimination has to be based on data collected by mobile teams and sentinel sites. Standard surveys based on sampling approaches are not appropriate for monitoring and evaluating trypanosomiasis elimination as they do not have sufficient statistical power for decision-making due to the low prevalence of the disease. The xenomonitoring of HAT infection in vectors could contribute to monitoring the elimination of sleeping sickness by assessing the presence or absence of parasites circulating in the vector. However it is not currently applicable since tests for molecular detection of $T$. b. gambiense infections in tsetse flies need to improve their sensitivity and specificity if reliable results are to be obtained. In addition, the low prevalence of infection in tsetse flies will require large-scale capture of flies and to develop protocols for pooling samples.
HAT will be considered eliminated in those foci where after adequate surveillance is in place, no cases are detected in all villages during a 5 -year period. The country will have to submit to the WHO a technical report about the elimination of the focus and request external evaluation by an international validation group (IVG). The validation system has to be able to ascertain the accessibility, coverage and quality of the surveillance activities to verify the absence of transmission and therefore to validate the elimination. The IVG will submit to the WHO a report with the appropriate recommendations concerning HAT elimination in the assessed focus. If the IVG evaluation is positive, the WHO will endorse elimination in the focus; otherwise, the WHO will recommend corrective measures and the way forward.

Even after a positive evaluation from the IVG, the surveillance system has to be maintained in sentinel sites to continue monitoring and evaluating the focus. Staff working in primary healthcare centres and staff in sentinel sites will receive regular refresher training to recognize the signs and symptoms suggestive of gambiense HAT, and to maintain awareness of the disease. The duration of this surveillance and preparedness for response has yet to be determined (World Health Organization, 2012b).

\section{INDICATORS AND MILESTONES}

In the framework of HAT elimination, the initial aim is the reduction of HAT incidence to fewer than 1 case per 10000 inhabitants in at least $90 \%$ of endemic areas, and to fewer than 2000 new cases reported globally by 2020 (Maurice, 2013). The final aim is elimination as defined by a reduction of HAT incidence to no new cases from any foci by 2030 (World Health Organization, 2012b).

The progress towards elimination will be measured by two primary quantitative indicators that will be updated annually:

1. The number of new cases of HAT reported.

2. The number of foci validated as eliminated.

Two secondary sets of indicators are proposed to assess the quality of the primary indicators and the scope of the elimination activities.

1. Proportion of the population at risk covered by control and surveillance activities (including active and passive screening) (Simarro et al. $2013 b)$. This indicator will be updated yearly.

2. Geographical extent of the infection and population at different levels of risk, as measured with a previously described method (Simarro et al. 2012). This indicator will be calculated every 2 years. 
Table 2. Quantitative indicators for HAT elimination as public health problem, 2012-2020 (World Health Organization, 2012b)

\begin{tabular}{llllllllll}
\hline \hline & 2012 & 2013 & 2014 & 2015 & 2016 & 2017 & 2018 & 2019 & 2020 \\
\hline $\begin{array}{l}\text { Cases annually reported } \\
\begin{array}{l}\text { Number of foci reporting fewer than 1 case } \\
\text { per 10000 inhabitants }\end{array}\end{array}$ & 6000 & 5500 & 5000 & 4500 & 4000 & 3500 & 3000 & 2500 & $<2000$ \\
$80 \%$ & $>90 \%$ \\
\hline
\end{tabular}

The milestones defined for HAT in the WHO Roadmap on NTDs and agreed by the SSNCPs are shown in Table 2.

ROADBLOCKS TO BE OVERCOME AND RESEARCH GAPS AND TOOLS NEEDED

On the road to eliminate HAT there exist important challenges that need to be overcome. These challenges need to be addressed if gambiense HAT elimination is to be achieved. They can be classified in different groups:

\section{Epidemiological knowledge of the disease}

Knowledge gaps in geographical distribution. Grey areas are still present, where epidemiological knowledge is limited. These are found mainly in old foci without appropriate surveillance, and in regions characterized by difficult accessibility, or affected by security constraints. The epidemiological situation in these areas needs to be clarified. Adapted and adequate sensitive tools will help to assess these areas and to fill the mapping gaps.

Epidemiological role of asymptomatic, seropositive human carriers with undetectable parasitaemia. The presence of seropositive/aparasitaemic human cases and healthy carriers is well described in gambiense HAT (Bucheton et al. 2011; Jamonneau et al. 2012). They could play a role in the persistence of disease transmission. Further studies are needed to clarify their epidemiological significance, and to develop appropriate strategies for their management.

Epidemiological role of animals as reservoir of gambiense HAT. The presence of T. b. gambiense in animals has been widely described (Duke, 1931; Makumyaviri et al. 1989; Njiokou et al. 2006, 2010; Cordon-Obras et al. 2009). The role they can play as reservoirs to maintain the transmission of gambiense HAT needs to be elucidated.

Underdetection and underreporting. Gambiense HAT is a rural disease, occurring in remote areas not always fully accessible for the health system. Under-detection and under-reporting are a reality, and they have been estimated in different ways.
Additional indicators and modelling tools for estimating the location and abundance of undetected cases have to be developed.

Risk of reintroduction of the infection after elimination from a focus. A risk of reintroduction of HAT after elimination exists, and it requires maintenance of surveillance activities after HAT has been eliminated from a focus. Studies on the risk factors of HAT reintroduction are encouraged, with a view to ensuring sustainability of elimination.

Integration of control and surveillance into existing health systems. Health systems in gambiense HAT endemic areas are often characterized by low attendance rates at healthcare facilities, lack of preparedness of staff and sometimes staff overloading. Operational research is needed to improve the integration of control and surveillance of gambiense HAT into the existing health systems.

Lack of skills of health staff on gambiense HAT control and surveillance. Experienced and committed staff presently involved in HAT control and surveillance are progressively retiring, and perspectives for appropriate replacement are limited. Approaches to engage a new generation of health staff on gambiense HAT elimination need to be implemented.

Lack of awareness among people at risk. When the disease becomes rare, local knowledge of the disease among the affected populations is progressively lost. Campaigns and tools to maintain awareness of gambiense HAT among the populations in endemic areas have to be developed and implemented.

Xenomonitoring for assessing gambiense HAT elimination. The feasibility of monitoring T.b. gambiense infections in vectors should be further explored as a potential tool for assessing HAT elimination programmes.

\section{Screening, diagnosis and staging}

Simpler and ensured serological tests. The introduction of simple, individual serological tests can help the integration of gambiense HAT control and surveillance into the health system, which will be critical to achieve the elimination goal. Individual 
serological tests are already under evaluation for gambiense HAT. The available screening tools (CATT and individual screening tests) are based on the same native antigen which has a ceiling in its production. New screening tools using new recombinant antigens which are easier to produce are needed.

Accurate and simpler parasitological confirmation tests. The current diagnostic tools to confirm the disease are complex and tedious, have a limited sensitivity and they need skilled and experienced staff. More user-friendly diagnostic tests for confirmation are needed to spread the diagnostic capabilities to the health services.

Less-invasive staging tests. The staging of HAT to establish the appropriate treatment is based on the analysis of CSF obtained from a lumbar puncture. This is a painful and not risk-free procedure. Development of tests on blood or urine for stage determination are encouraged.

\section{Treatment}

New user-friendly treatments. The current therapeutic regimens are complex to apply, cumbersome in their logistics for distribution, and characterized by considerable safety concerns. Safe drugs - if possible oral - which are easy to use and active against both forms and both stages of the disease are required.

Clinical trials. Clinical trials to develop new drugs for HAT are complex and long lasting. The reduction in the number of patients as elimination progresses will further complicate clinical trials. Efforts to facilitate clinical trials, always ensuring the safety of the patients, have to be considered.

\section{CONCLUSIONS}

The elimination of gambiense HAT is feasible, as a result of the epidemiological vulnerability of the disease, the current state of disease control, the availability of effective strategies and tools, the existence of international commitment and political will. The available strategies and tools have to be adapted to the different epidemiological settings to maximize cost-effectiveness.

Basic and operational research is needed to address knowledge gaps and to improve control tools. The development of new diagnostic and treatment tools will enhance control interventions and will facilitate the integration of a sensitive surveillance into the health systems. The strategies for elimination should be monitored and evaluated to ensure their appropriateness and effectiveness in an elimination scenario, to identify optimal interventions, and to establish the timing for switching from one strategy to the next.

The successful control activities carried out over the last 15 years have substantially reduced the burden of disease. Donors tend to lose interest when the burden of a disease is decreasing. To eliminate gambiense HAT a long-term commitment by donors is needed. Advocacy is needed to sensitize donors on the importance of switching from a control to an elimination mindset. Adequate funding must be made available not only to implement elimination activities in the field, but also to support research that will make the elimination effort sustainable.

Alongside the commitment of donors, ownership of the objectives and process of elimination by endemic countries is critical. Gambiense HAT elimination has to be included in national health plans to facilitate its prioritization and to maintain its profile against competing health priorities.

The integration of gambiense HAT control and surveillance programmes in health systems is a major challenge. In the rural areas where trypanosomiasis is prevalent, the overall performance of peripheral health systems is often weak and characterized by unskilled staff, low attendance and low coverage. Therefore, the strengthening of health systems to implement the activities included in the elimination strategies is essential.

Political instability and insecurity are inescapable challenges in an elimination programme. The successful elimination of gambiense HAT requires a turmoil-free socio-political context.

Past and current experiences show that HAT elimination is difficult and will require more effort, time, and money than initially anticipated. The inherent risks of failure are offset by the benefits that accrue from a successful elimination campaign. An elimination campaign has the ability to inspire dedication and sacrifice among health workers, and to attract donors, both of which are needed to overcome the hurdles to success (Hopkins, 2013).

Much progress has been made in the fight against gambiense HAT, but the final line of the struggle is not in sight just yet.

\section{ACKNOWLEDGEMENTS}

The authors would like to thank the coordinators of the different National Sleeping Sickness Control Programmes, the staff of the HAT WHO collaborating centres and the members of the WHO Expert Advisory Panel on Trypanosomiasis for their participation in the fruitful discussions which were the base of this paper. Special acknowledgement to Giuliano Cecchi and Massimo Paone for the critical reading of the document and for their technical support in the framework of the collaboration between the WHO and the FAO (Food and Agriculture Organization of the United Nations) through the Programme against African Trypanosomosis (PAAT). 


\section{REFERENCES}

Brun, R., Blum, J., Chappuis, F. and Burri, C. (2010). Human African trypanosomiasis. Lancet $\mathbf{3 7 5}, 148-159$

Bruto da Costa, B. F., Sant'Anna, J.F., Correia dos Santos, A., de Araujo Alvares, M. G. and Wyllie, J. A. (1916). Sleeping Sickness; a Record of Four Years' War Against it in Principe, Portuguese West Africa. Published for the Centro colonial, Lisbon, by Ballière, Tindall and Cox, London, UK.

Bucheton, B., MacLeod, A. and Jamonneau, V. (2011). Human host determinants influencing the outcome of Trypanosoma brucei gambiense infections. Parasite Immunology 33, 438-447.

Buscher, P., Gilleman, Q. and Lejon, V. (2013). Rapid diagnostic test for sleeping sickness. New England fournal of Medicine 368, 1069-1070. Centers for Disease Control and Prevention (CDC) (1993). Recommendations of the International Task Force for Disease Eradication. Morbidity and Mortality Weekly Report 42, No. RR-16.

Checchi, F., Filipe, J.A., Haydon, D. T., Chandramohan, D. and Chappuis, F. (2008). Estimates of the duration of the early and late stage of gambiense sleeping sickness. BMC Infectious Diseases 8, 16.

Checchi, F., Cox, A. P., Chappuis, F., Priotto, G., Chandramohan, D. and Haydon, D. T. (2012). Prevalence and under-detection of gambiense human African trypanosomiasis during mass screening sessions in Uganda and Sudan. Parasites and Vectors 5, 157.

Cordon-Obras, C., Berzosa, P., Ndong-Mabale, N., Bobuakasi, L., Buatiche, J. N., Ndongo-Asumu, P., Benito, A. and Cano, J. (2009). Trypanosoma brucei gambiense in domestic livestock of Kogo and Mbini foci (Equatorial Guinea). Tropical Medicine and International Health 14, 535-541.

Duke, H. L. (1931). Trypanosoma gambiense in monkeys and ruminants; prolonged infection, immunity, and superinfection. Parasitology 23, 325-345

Esterhuizen, J., Rayaisse, J. B., Tirados, I., Mpiana, S., Solano, P., Vale, G. A., Lehane, M. J. and Torr, S. J. (2011). Improving the costeffectiveness of visual devices for the control of riverine tsetse flies, the major vectors of human African trypanosomiasis. PLoS Neglected Tropical Diseases 5, e1257.

Fèvre, E. M., Coleman, P. G., Odiit, M., Magona, J. W., Welburn, S. C. and Woolhouse, M. E. J. (2001). The origins of a new Trypanosoma brucei rhodesiense sleeping sickness outbreak in eastern Uganda. Lancet 358, $625-628$

Fèvre, E. M., Coleman, P. G., Welburn, S. C. and Maudlin, I. (2004). Reanalyzing the 1900-1920 sleeping sickness epidemic in Uganda. Emerging Infectious Diseases 10, 567-573.

Funk, S., Nishiura, H., Heesterbeek, H., Edmunds, W. J. and Checchi, F. (2013). Identifying transmission cycles at the human-animal interface: the role of animal reservoirs in maintaining Gambiense human African trypanosomiasis. PLoS Computational Biology 9, e1002855. doi: 10.1371/journal.pcbi.1002855

Hargrove, J.W. (2002). Tsetse Eradication: Sufficiency, Necessity and Desirability. DFID Animal Health Programme. Centre for Tropical Veterinary Medicine, University of Edinburgh, Edinburgh, UK

Hide, G. (1999). History of sleeping sickness in East Africa. Clinical Microbiology Reviews 12, 112-125.

Hide, G., Tait, A., Maudlin, I. and Welburn, S. C. (1996). The origins, dynamics and generation of Trypanosoma brucei rhodesiense epidemics in East Africa. Parasitology Today 12, 50-55.

Hopkins, D. R. (2013). Disease eradication. New England Fournal of Medicine 368, 54-63.

Jacobs, R. T., Nare, B., Wring, S. A., Orr, M. D., Chen, D., Sligar, J. M., Jenks, M.X., Noe, R. A., Bowling, T.S., Mercer, L. T., Rewerts, C., Gaukel, E., Owens, J., Parham, R., Randolph, R., Beaudet, B., Bacchi, C. J., Yarlett, N., Plattner, J.J., Freund, Y., Ding, C., Akama, T., Zhang, Y.-K., Brun, R., Kaiser, M., Scandale, I. and Don, R. (2011). SCYX-7158, an orally-active benzoxaborole for the treatment of stage 2 human African trypanosomiasis. PLoS Neglected Tropical Diseases 5, e1151. doi: 10.1371/journal.pntd.0001151

Jamonneau, V., Bucheton, B., Kabore, J., Ilboudo, H., Camara, O., Courtin, F., Solano, P., Kaba, D., Kambire, R., Lingue, K., Camara, M., Baelmans, R., Lejon, V. and Büscher, P. (2010). Revisiting the immune trypanolysis test to optimize epidemiological surveillance and control of sleeping sickness in West Africa. PLoS Neglected Tropical Diseases 4, e917. doi: 10.1371/journal.pntd.0000917.

Jamonneau, V., Ilboudo, H., Kabore, J., Kaba, D., Koffi, M., Solano, P., Garcia, A., Courtin, D., Laveissière, C., Lingue, K., Büscher, P. and Bucheton, B. (2012). Untreated human infections by Trypanosoma brucei gambiense are not $100 \%$ fatal. PLoS Neglected Tropical Diseases 6, e1691. doi: 10.1371/journal.pntd.0001691.
Jelinek, T., Bisoffi, Z., Bonazzi, L., van Thiel, P., Bronner, U., de Frey, A., Gundersen, S. G., McWhinney, P., Ripamonti, D. and European Network on Imported Infectious Disease Surveillance (2002). Cluster of African trypanosomiasis in travellers to Tanzanian national parks. Emerging Infectious Diseases 8, 634-635. doi: 10.3201/eid0806.010432.

Kaare, M. T., Picozzi, K., Mlengeya, T., Fèvre, E. M., Mellau, L. S. Mtambo, M. M., Cleaveland, S. and Welburn, S. C. (2007). Sleeping sickness - a re-emerging disease in the Serengeti? Travel Medicine and Infectious Disease 5, 117-124.

Kennedy, P. G. (2008). The continuing problem of human African trypanosomiasis (sleeping sickness). Annals of Neurology 64, 116-126.

Lutumba, P., Makieya, E., Shaw, A., Meheus, F. and Boelaert, M. (2007). Human African trypanosomiasis in a rural community, Democratic Republic of Congo. Emerging Infectious Diseases 13, 248-254.

Makumyaviri, A., Mehlitz, D., Kageruka, P., Kazyumba, G. L. and Molisho, D. (1989). Animal reservoir hosts of Trypanosoma brucei gambiense in Zaire: trypanosome infections in two foci in Bas-Zaire. Tropical Medicine and Parasitology 40, 258-262.

Maurice, J. (2013). New WHO plan targets the demise of sleeping sickness. Lancet 381, 13-14

Molyneux, D. H. (2001). Sterile insect release and trypanosomiasis control: a plea for realism. Trends in Parasitology 17, 413-414.

Morris, K. R.S. (1959). The epidemiology of sleeping sickness in East Africa. I. A sleeping sickness outbreak in Uganda in 1957. Transactions of the Royal Society of Tropical Medicine and Hygiene 53, 384-393.

Mumba, D., Bohorquez, E., Messina, J., Kande, V., Taylor, S. M., Tshefu, A. K., Muwonga, J., Kashamuka, M. M., Emch, M., Tidwell, R., Büscher, P. and Meshnick, S. R. (2011). Prevalence of human African trypanosomiasis in the Democratic Republic of the Congo. PLoS Neglected Tropical Diseases 5, e1246. doi: 10.1371/journal. pntd.0001246.

Njiokou, F., Laveissiere, C., Simo, G., Nkinin, S., Grébaut, P., Cuny, G. and Herder, S. (2006). Wild fauna as a probable animal reservoir for Trypanosoma brucei gambiense in Cameroon. Infection Genetics Evolution 6, 147-153.

Njiokou, F., Nimpaye, H., Simo, G., Njitchouang, G. R. Asonganyi, T., Cuny, G. and Herder, S. (2010). Domestic animals as potential reservoir hosts of Trypanosoma brucei gambiense in sleeping sickness foci in Cameroon. Parasite 17, 61-66.

Organization of African Unity (2001). Pan African Tsetse and Trypanosomosis Eradication Campaign (PATTEC). Enhancing Africa's Prosperity. Plan of action. Addis Ababa, Ethiopia.

Robays, J., Bilengue, M. C. C., Van der Stuyft, P. and Boelaert, M. (2004). The effectiveness of active population screening and treatment for sleeping sickness control in the Democratic Republic of Congo. Tropical Medicine and International Health 9, 542-550. doi: 10.1111/j.13653156.2004.01240.x.

Selby, R., Bardosh, K., Picozzi, K., Waiswa, C. and Welburn, S. C. (2013). Cattle movements and trypanosomes: restocking efforts and the spread of Trypanosoma brucei rhodesiense sleeping sickness in post-conflict Uganda. Parasites and Vectors 6, 281.

Simarro, P. P., Franco, J. R., Ndongo, P., Nguema, E., Louis, F. J. and Jannin, J. (2006). The elimination of Trypanosoma brucei gambiense sleeping sickness in the focus of Luba, Bioko Island, Equatorial Guinea. Tropical Medicine and International Health 11, 36-46.

Simarro, P.P., Jannin, J. and Cattand, P. (2008). Eliminating human African trypanosomiasis: where do we stand and what comes next? PLoS Medicine 5, 174-180.

Simarro, P.P., Cecchi, G., Paone, M., Franco, J. R., Diarra, A., Ruiz, J. A., Fèvre, E. M., Courtin, F., Mattioli, R. C. and Jannin, J. G. (2010). The atlas of human African trypanosomiasis: a contribution to global mapping of neglected tropical diseases. International fournal of Health Geographics 9, 57.

Simarro, P. P., Diarra, A., Postigo, J. A. R., Franco, J. R. and Jannin, J. G. (2011). The human African trypanosomiasis control and surveillance programme of the World Health Organization 2000-2009: the way forward. PLoS Neglected Tropical Diseases 5, e1007. doi: 10.1371/ journal.pntd.0001007.

Simarro, P.P., Cecchi, G., Franco, J. R., Paone, M., Diarra, A., Ruiz-Postigo, J. A., Fèvre, E. M., Mattioli, R. C. and Jannin, J. G. (2012). Estimating and mapping the population at risk of sleeping sickness. PLoS Neglected Tropical Diseases 6, e1859. doi: 10.1371/journal. pntd.0001859

Simarro, P.P., Franco, J. R., Diarra, A., Ruiz-Postigo, J. A. and Jannin, J. G. (2013a). Diversity of human African trypanosomiasis epidemiological settings requires fine-tuning control strategies to facilitate disease elimination. Research and Reports in Tropical Medicine 4, 1-6. 
Simarro, P.P., Cecchi, G., Franco, J.R., Paone, M., Diarra, A., Ruiz-Postigo, J. A., Mattioli, R. C. and Jannin, J. G. (2013b). Mapping the capacities of fixed health facilities to cover people at risk of gambiense human African trypanosomiasis. International Fournal of Health Geographics (in press).

Solano, P., Torr, S. J. and Lehane, M. (2013). Is vector control needed to eliminate gambiense human African trypanosomiasis? Frontiers in Cellular and Infection Microbiology 3, 33. doi: 10.3389/fcimb.2013.00033.

Steverding, D. (2008). The history of African trypanosomiasis. Parasites and Vectors 1, 3. doi: 10.1186/1756-3305-1-3.

Torreele, E., Bourdin Trunz, B., Tweats, D., Kaiser, M., Brun, R. Mazué, G., Bray, M. A. and Pécoul, B. (2010). Fexinidazole - a new oral nitroimidazole drug candidate entering clinical development for the treatment of sleeping sickness. PLoS Neglected Tropical Diseases 4, e923. doi: 10.1371/journal.pntd.0000923.

Van Meirvenne, N., Magnus, E. and Buscher, P. (1995). Evaluation of variant specific trypanolysis tests for serodiagnosis of human infections with Trypanosoma brucei gambiense. Acta Tropica 60, 189-199.

Welburn, S. C. and Maudlin, I. (2012). Priorities for the elimination of sleeping sickness. Advances in Parasitology 79, 299-337.

Welburn, S. C., Maudlin, I. and Simarro, P.P. (2009). Controlling sleeping sickness - a review. Parasitology 136, 1943-1949. doi: 10.1017/ S0031182009006416.

World Health Organization (1986). Epidemiology and Control of African Trypanosomiasis. Report of a WHO Expert Committee. WHO Technical Report Series. No 739. World Health Organization, Geneva, Switzerland. World Health Organization (1997). 50th World Health Assembly Resolutions. World Health Organization, Geneva, Switzerland. http:/ www.who.int/neglected_diseases/mediacentre/WHA_50.36_Eng.pdf.

World Health Organization (1998). Control and Surveillance of African Trypanosomiasis. WHO Technical Report Series. No 881. World Health Organization, Geneva, Switzerland.
World Health Organization (2000). Report on Global Surveillance of Epidemic-prone Infectious Diseases. World Health Organization, Geneva, Switzerland. http://whqlibdoc.who.int/hq/2000/WHO_CDS_CSR_ISR 2000.1.pdf.

World Health Organization (2003). 56th World Health Assembly Resolutions. World Health Organization, Geneva, Switzerland. http:// www.who.int/neglected_diseases/mediacentre/WHA_56.7_Eng.pdf.

World Health Organization (2004). 57th World Health Assembly Resolutions. World Health Organization, Geneva, Switzerland. http:// www.who.int/neglected_diseases/mediacentre/WHA_57.2_Eng.pdf.

World Health Organization (2006). Human African trypanosomiasis (sleeping sickness): epidemiological update. Weekly Epidemiological Record 81, 71-80.

World Health Organization (2012a). Accelerating Work to Overcome the Global Impact of Neglected Tropical Diseases - A Roadmap for Implementation. WHO/HTM/NTD/ 2012.1. World Health Organization, Geneva, Switzerland.

World Health Organization (2012b). Report of a WHO Meeting on Elimination of African Trypanosomiasis (Trypanosoma brucei gambiense). Geneva, 3-5 December 2012. WHO/HTM/NTD/IDM 2013.4. World Health Organization, Geneva, Switzerland. http://apps who.int/iris/bitstream/10665/79689/1/WHO_HTM_NTD_IDM_2013.4 eng.pdf.

World Health Organization (2013a). London Declaration on Neglected Tropical Diseases. World Health Organization, Geneva, Switzerland. http:// www.who.int/neglected_diseases/London_Declaration_NTDs.pdf.

World Health Organization (2013b). 66th World Health Assembly Resolutions. World Health Organization, Geneva, Switzerland. http:// www.who.int/neglected_diseases/mediacentre/WHA_66.12_Eng.pdf.

World Health Organization (2013c). Control and Surveillance of African Trypanosomiasis. WHO Technical Report Series. No 984. World Health Organization, Geneva, Switzerland 